\title{
Selective modification for polydimethylsiloxane chip by micro-plasma
}

\author{
Jiaxing Li $\cdot$ Xiang Wang $\cdot$ Cheng Cheng $\cdot$ \\ Limu Wang $\cdot$ Eric Zhao $\cdot$ Xiangke Wang . \\ Weijia Wen
}

Received: 30 July 2012/Accepted: 6 September 2012/Published online: 21 September 2012

(C) Springer Science+Business Media, LLC 2012

\begin{abstract}
We report a method to selectively modify polydimethylsiloxane (PDMS) chip in a fast and facile way using micro-plasma approach in the atmospheric-pressure. Pure $\mathrm{He}$ and $\mathrm{He} /$ acrylic acid plasma were ignited directly in different channels of PDMS microchip. Our experiments results yielded strong hydrophilic property on the surface of PDMS by the plasma treatment.
\end{abstract}

\section{Introduction}

Chemical and physical modifications of the surface of microfluidic channel is a state-of-the-art technique to control the surface charge and hydrophilicity in the micro analysis system [1-9]. Micro-plasma technique has received special attention due to its application in surface modification as well as on-chip light sources and chromatography detectors $[1,10]$. Herein, we demonstrate the use of He/acrylic acid (AA) atmospheric-pressure micro-plasmas in a polydimethyl siloxane (PDMS) microchip to selectively control the surface hydrophilicity of PDMS.

PDMS is one of the most popular materials in microfluidics for its superior advantages of easy fabrication, low cost and disposability [11]. However, the extremely hydrophobic property of PDMS surface limits the extent of its

J. Li $\cdot$ X. Wang $\cdot$ L. Wang $\cdot$ E. Zhao $\cdot$ W. Wen $(\bowtie)$

Department of Physics, The Hong Kong University of Science and Technology, Clear Water Bay, Kowloon, Hong Kong e-mail: phwen@ust.hk

J. Li $\cdot$ C. Cheng $\cdot$ X. Wang $(\bowtie)$

Key Laboratory of Novel Thin Film Solar Cells, Institute of Plasma Physics, Chinese Academy of Sciences, P.O. Box 1126, Hefei 230031, People's Republic of China

e-mail: xkwang@ipp.ac.cn applications. As a result, many different methods for hydrophilization have been proposed to modify the surface of PDMS, such as rendering it hydrophilic by activating PDMS surface using oxygen plasma [9] and absorbed hydrophilic organic molecules coatings [8, 12], nonetheless at the expense of its long-term stability [3]. Another conventional way of surface modification in polymer science is surface-attached polymerization, which provides chemically stable surfaces due to the covalent bonding of the polymer chains to the substrate [4-6,9]. Recent reports also introduce grafting of poly (acrylic acid) (PAA) on PDMS based on a liquid phase system with UV irradiation [4, 5, 13, 14], however such method demands long operation hours, and requires strictly experimental conditions and abundant chemicals as well, some result in PDMS swelling in organic solvents, and the others poor stability and performance. The micro-plasma technique with working gas $\mathrm{He}$ and AA presented in this work circumvents the above drawbacks, owing to its selectivity, quickness and convenience.

\section{Experimental}

PDMS (Sylgard 184 Silicone Elastomer, Dow Corning Corporation), employed as the polymeric matrix, was acquired in the forms of a pre-polymer and a cross-linker. Acrylic acid (AA, $99 \%$ ) was purchased from SigmaAldrich. He (99.9\%) was purchased from Hefei Hengshun Gas Company.

Figure 1a shows atmospheric-pressure plasma device for grafting PAA on the surface of PDMS microchip. One electrode is the stainless steel tubing and another is the metal wire. Firstly, He, the working gas, was introduced in microchip channel by a flow meter $(1-5 \mathrm{~mL} / \mathrm{min})$ and ignited by the $\mathrm{AC}$ power (frequency is chosen in the range 
Fig. 1 a Schematic diagram of experimental setup and b reaction mechanism

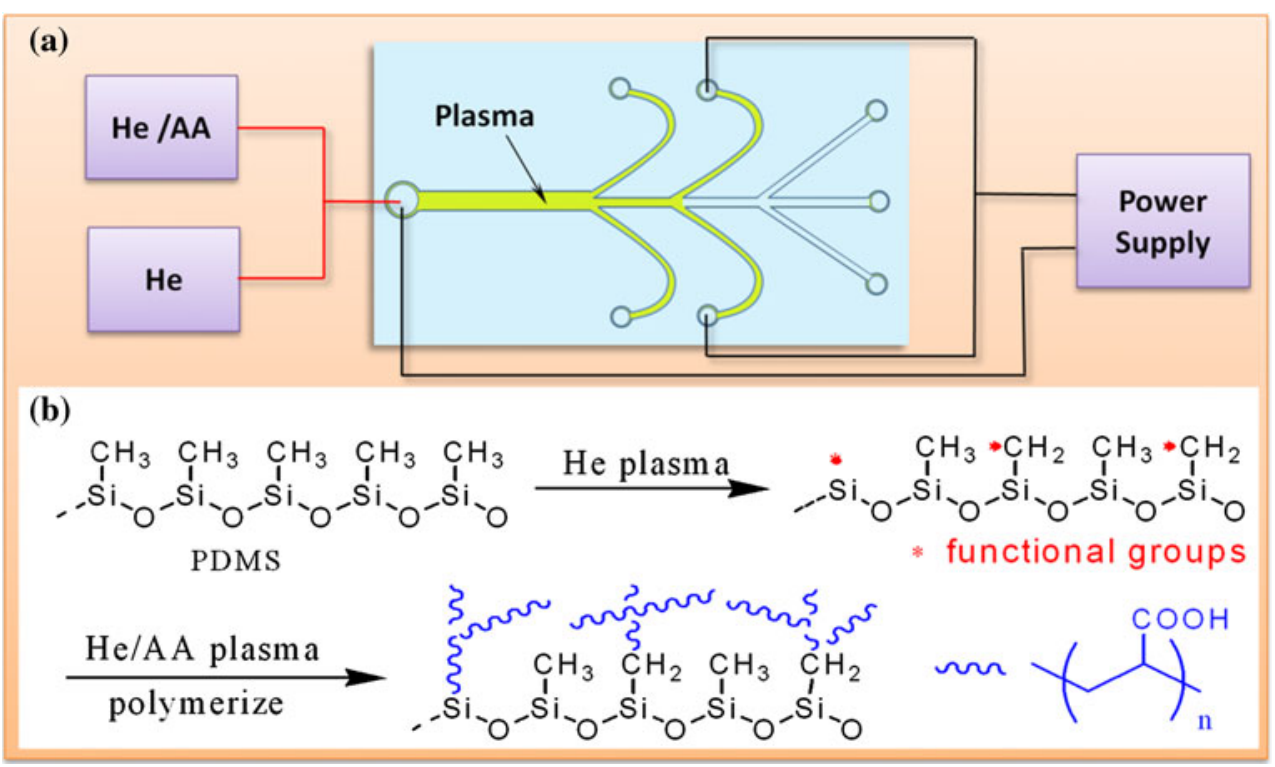

of $6-40 \mathrm{kHz}$ ) for stable discharge for $1 \mathrm{~min}$. The He/AA mixture gas (AA-saturated $\mathrm{He}$ ) was then loaded into microchip with the same rate and was ignited. The channel was treated for about $10 \mathrm{~min}$.

The morphologies of the untreated and grafted AA PDMS surface were observed by SEM (JEOL-6700F) with an acceleration voltage of $15 \mathrm{kV}$. The FT-IR transmission spectrum was recorded on a Bio-Rad FTS6000 spectrometer with a DTGS detector. We recorded and measured the droplets in different microchannels by optical microscope (Olympus 1X71, Olympus Imaging America Inc., PA, USA) with accessorial CCD.

\section{Results and discussion}

Figure $1 b$ illustrates the possible reaction mechanism for the modification of PDMS surface. When He/AA plasma was permeated in the channel for a preset time frame, the active AA molecules in the plasma environment would react with the functional groups (such as methylene radicals) on the surface of PDMS and polymerize into a PAA chain. After He/AA plasma treatment, a large number of carboxyl groups were grafted on the PDMS turning the formerly hydrophobic surface into a completely hydrophilic surface.

Figure 2 depicts the voltage and current waveform corresponding to discharge in the linear microchip (Fig. 3a). When the pure He homogenous plasma was generated, the voltage and current maxima were about $3.85 \mathrm{kV}$ and $11.5 \mathrm{~mA}$ respectively. When $\mathrm{He}$, saturated with AA produced homogenous plasma as a working gas, the voltage and current were increased to $4.5 \mathrm{kV}$ and $15 \mathrm{~mA}$ accordingly, probably due to the electronegativity of the AA vapour.
Figure $3 \mathrm{a}-\mathrm{c}$ describes the states of different microchips under He/AA plasma treatment. We observed the plasma near electrode connected with high voltage on the channel of microchip with width $200 \mu \mathrm{m}$ and length $4.5 \mathrm{~cm}$ to be brighter than that with low voltage. The reason behind was that the plasma is similar to plasma bullet $[15,16]$. Since the length of channels with three or eight inlets (Fig. 3b, c) is shorter compared with that of straight channel, with appropriate voltage and frequency, well-distributed plasma can be generated in the former as well. In addition, by successfully introducing plasma in microchips with channels bent at $90^{\circ}$, renders the possibility of generating plasma in microchips with gentler channel bend. Figure 3b, c shows selective modification was achieved when electrodes were located at different inlets. Figure 3d, e display the modification effects of PDMS before and after plasma treatment, where the untreated microchip, only water-in-oil droplets formed while in modified microchips, oil-in-water droplets could be formed.

Surface structure plays an important role on the wettability of solid surface. As Fig. 4 reveals the surfaces of untreated PDMS and treated PDMS by pure He are uniformly flat while that of treated PDMS by He/AA plasma has rough microstructure, which increases the wettability of solid surface [4]. The pure He plasma did not damage the structure of PDMS surface. PAA was coated by He/AA plasma on the surface of PDMS and formed the rough surface with wavy morphology. The PDMS channel exhibits obvious hydrophilicity owing to the rough surface morphology and hydrophilic property of PAA. The resulting wettability contrast between treated areas and untreated areas of PDMS channel had been investigated as shown in the inserted images. As expected, the shape of the meniscus of oil-water interface clearly showed that oil is wetting the untreated PDMS outside the plasma treated area. 
Fig. 2 Voltage and current signals corresponding to the power of linear microchip

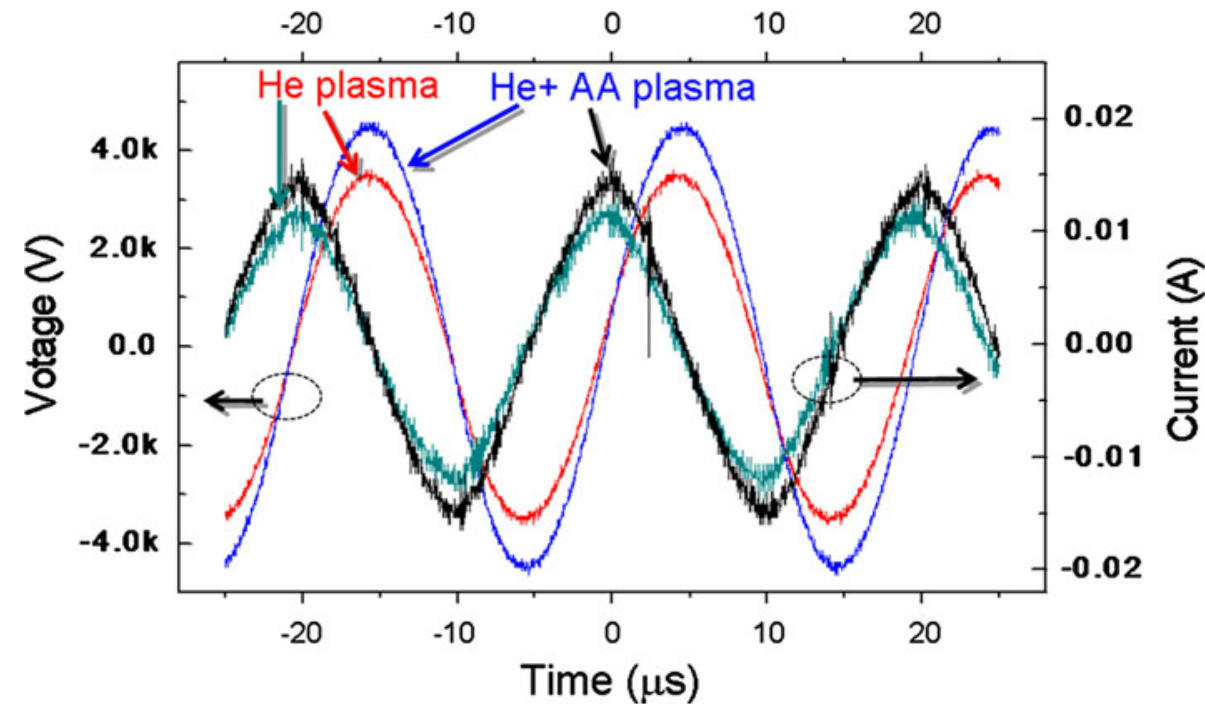

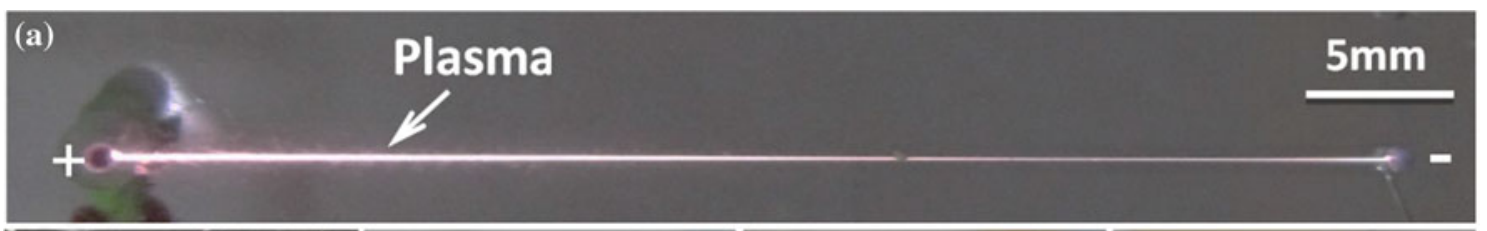
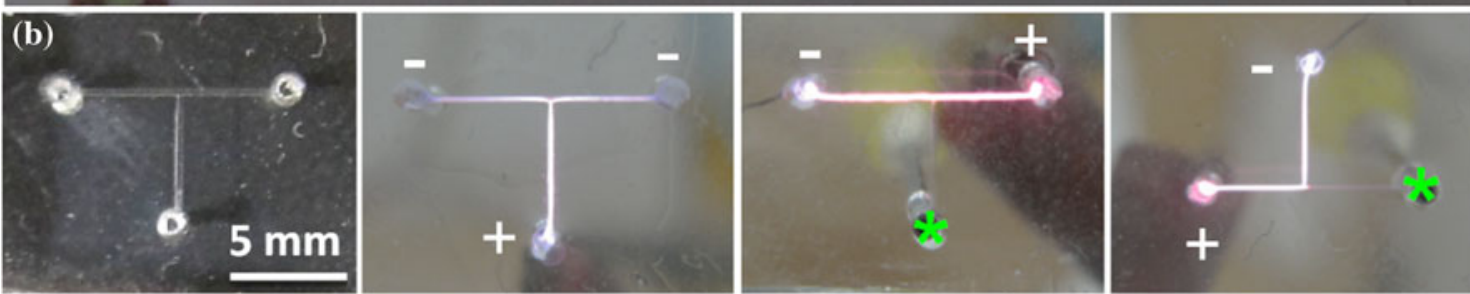

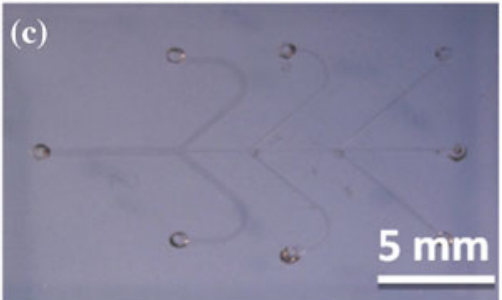

(d)

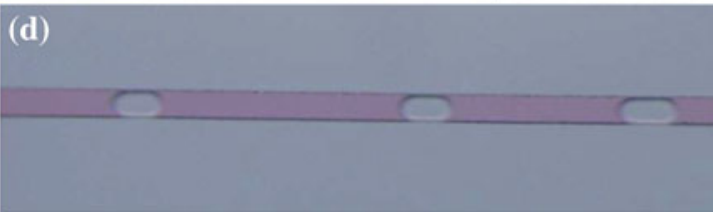

Fig. 3 a Microplasma in linear microchip, b joint microchip, c eightinlet microchip, the work gas is introduced from the inlet marked plus, plus indicates electrodes with high voltage, minus indicates

FT-IR detection was conducted to verify that the PDMS surface prepared by the present method was highly functionalized. Fig. 5 shows the FT-IR spectra of untreated PDMS and treated PDMS by He and He/AA (placed in a drying cabinet for over 1 month). The spectra of untreated PDMS and treated PDMS by He have areas of overlapping. The absorption band at $2950-2970 \mathrm{~cm}^{-1}$ and the band at $1245-1270 \mathrm{~cm}^{-1}$ electrodes with low voltage, the inlet marked with asterisk is sealed. d Water-in-oil droplet in untreated microchip, and e oil-in-water droplets in plasma-modified microchip

corresponded to asymmetric $-\mathrm{CH}_{3}$ stretching and the symmetric $-\mathrm{CH}_{3}$ deformation band in $\mathrm{Si}-\mathrm{CH}_{3}$ group respectively [4]. For modified PDMS sample, the broad absorption at $3350-2500 \mathrm{~cm}^{-1}$ and the sharp and intense band at 1725 $-1700 \mathrm{~cm}^{-1}$ were attributed to the $-\mathrm{OH}$ stretch and the $-\mathrm{C}=\mathrm{O}$ stretch in $\mathrm{COOH}$, respectively, which indicated the presence of hydrophilic carboxylic acid groups [4, 17]. 


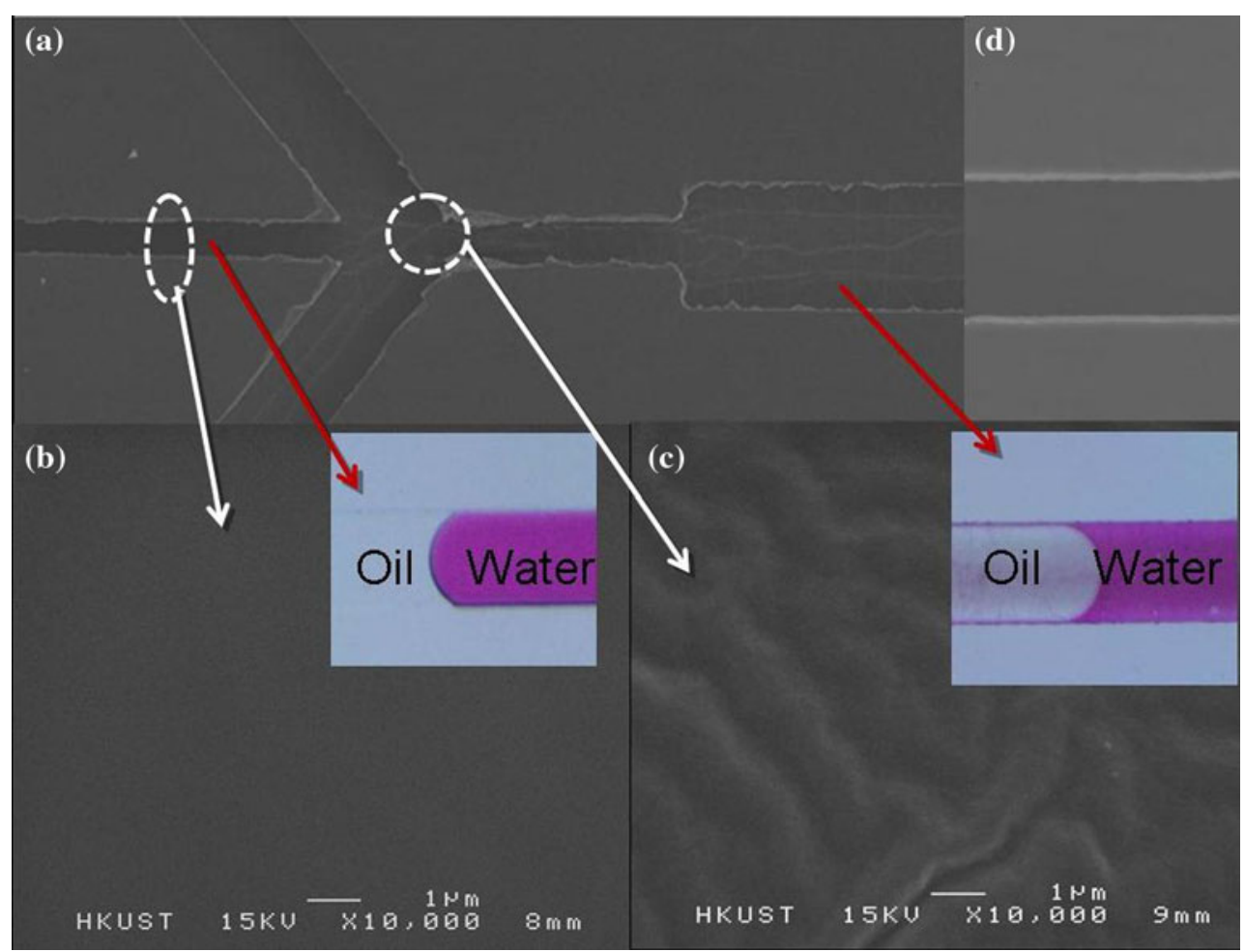

Fig. 4 a SEM images of microchannel, b surface of untreated microchannel, $\mathbf{c}$ surface of treated microchannel by He/AA. Contrast in wettability between treated and untreated parts and meniscus inversion inside treated zone, $\mathbf{d}$ surface of treated microchannel by pure He

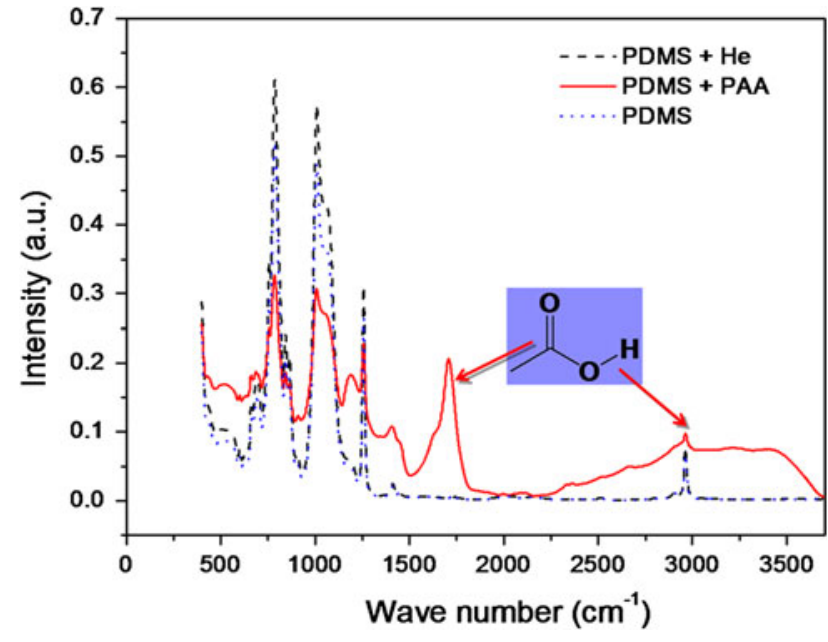

Fig. 5 FT-IR absorption spectra of native PDMS and treated PDMS by $\mathrm{He}$ and $\mathrm{He} / \mathrm{AA}$

\section{Conclusion}

In this work, we demonstrated the methodology of He/AA micro-plasma selective treatment on the PDMS surface to tune its hydrophilicity. By using such approach, the microfluidic channel surface can be easily modified for the different application purposes. It is expected that, other materials are possibly modified if the suitable gas monomers are selected.

Acknowledgements Authors Jiaxing Li and Xiang Wang contributed equally to this paper. The authors would like to acknowledge National Natural Science Foundation of China (21272236) and Hong Kong RGC grants HKUST 604710 for the financial support of this project.

\section{References}

1. Evju JK, Howell PB, Locascio LE, Tarlov MJ, Hickman JJ (2004) Appl Phys Lett 84:1668

2. Reyes DR, Iossifidis D, Auroux PA, Manz A (2002) Anal Chem $74: 2623$

3. Duffy DC, McDonald JC, Schueller OJA, Whitesides GM (1998) Anal Chem 70:4974

4. Schneider MH, Willaime H, Tran Y, Rezgui F, Tabeling P (2010) Anal Chem 82:8848

5. Schneider MH, Tran Y, Tabeling P (2011) Langmuir 27:1232

6. Lin BC, Wu DP, Qin JH (2007) Lab Chip 7:1490

7. Diaz-Quijada GA, Wayner DDM (2004) Langmuir 20:9607

8. Roman GT, Culbertson CT (2006) Langmuir 22:4445

9. Liu JK, Lee ML (2006) Electrophoresis 27:3533

10. Priest C, Gruner PJ, Szili EJ, Al-Bataineh SA, Bradley JW, Ralston J, Steele DA, Short RD (2011) Lab Chip 11:541

11. McDonald JC, Whitesides GM (2002) Accounts Chem Res $35: 491$ 
12. Wu DP, Luo Y, Zhou XM, Dai ZP, Lin BC (2005) Electrophoresis $26: 211$

13. $\mathrm{Hu} \mathrm{SW}$, Ren XQ, Bachman M, Sims CE, Li GP, Allbritton N (2002) Anal Chem 74:4117

14. Hu SW, Ren XQ, Bachman M, Sims CE, Li GP, Allbritton NL (2004) Anal Chem 76:1865
15. Chung TH, Kim SJ, Bae SH (2010) Phys Plasmas 17:053504

16. Shi JJ, Zhong FC, Zhang J, Liu DW, Kong MG (2008) Phys Plasmas 15:013504

17. Jiang ZQ, Jiang ZJ, Shi YC, Meng YD (2010) Appl Surf Sci 256:6473 